\title{
INTERPOLATION TO SOME CLASSES OF ANALYTIC FUNCTIONS BY FUNCTIONS WITH PRE-ASSIGNED POLES
}

\author{
BY \\ YU-CHENG SHEN
}

1. Introduction. Let $f(z)$ be a function analytic in the (open) interior $K$ of the unit circle $|z|=1$. Then $f(z)$ will be called a function of class( $\left.{ }^{1}\right) S_{\alpha}$, if there exists a real number $\alpha, \alpha>1$, such that the integral

$$
\mathfrak{M}_{\alpha}(f ; r)=\frac{\alpha-1}{\pi} \int_{0}^{r} \int_{0}^{2 \pi}\left(1-r^{2}\right)^{\alpha-2} \mid f\left(r e^{i \theta}\right){ }^{2} r d \theta d r
$$

is bounded for $0<r<1$. Since $\alpha>\beta>1$ implies

$$
\mathfrak{M}_{\alpha}(f ; r) \leqq \frac{\alpha-1}{\beta-1} \mathfrak{M}_{\beta}(f ; r),
$$

class $S_{\beta}$ is contained in class $S_{\alpha}$ for every $\alpha>\beta$. The right-hand side of (1.1), when expanded, is a function of $r^{2}$ (cf. §2). It is convenient to set $\rho=r^{2}$, and consider $\mathfrak{M}_{\alpha}(f ; \rho)$. This will be done in $\$ 2$.

Let $a_{n k}, k=1,2, \cdots, n ; n=1,2, \cdots$, be a set of points preassigned on $K$ and having no limit point on $K$. Two sets $a_{n k}$ and $b_{n k}$ will be regarded as equivalent if they are related to each other by a transformation of the form

$$
\zeta=\lambda \frac{z-c}{1-\bar{c} z}, \quad|\lambda|=1,|c|<1,
$$

that is, by a transformation which maps $|z| \leqq 1$ conformally onto $|\zeta| \leqq 1$ so. that $z=c$ corresponds to $\zeta=0$. To each point $a_{n k}$ will be assigned an order as follows: $a_{n k}$ is of order $m_{k}$ if exactly $m_{k}$ points in the set $\left(a_{n 1}, a_{n 2}, \cdots, a_{n, k-1}\right)$ coincide with $a_{n k}$.

Let $\delta_{k}$ denote the operation of differentiating $m_{k}$ times with respect to the parameter $a_{n k}$ followed by evaluation at the preassigned point $a_{n k}$. Let $\bar{\delta}_{k}$ denote the corresponding operation with respect to $\bar{a}_{n k}$. If $m_{k}=0$, we define $\delta_{k}=\bar{\delta}_{k}=1$.

Now let $a_{n k},\left|a_{n k}\right|<1$, and $\alpha, \alpha>1$, be given, and let the functions

$$
\phi_{n k}(z)=1 /\left(1-\bar{a}_{n k} z\right)^{\alpha}
$$

Presented to the Society, April 26, 1947; received by the editors October 18, 1946.

(1) These classes are among the classes treated by G. H. Hardy in connection with derivatives of fractional orders. Because Hardy's work has been inaccessible to the present writer since the war, general properties of the functions considered here are discussed in $\$ 2$. 
be defined by the infinite series

$$
1+\alpha \bar{a}_{n k} z+\frac{\alpha(\alpha+1)}{1 \cdot 2}\left(\bar{a}_{n k} z\right)^{2}+\cdots, \quad|z| \leqq 1 .
$$

Let $f(z)$ be an arbitrary function of class $S_{\alpha}$, and let $f_{n}(z)$ denote the function of the form

$$
f_{n}(z)=\sum_{k=1}^{n} A_{n k} \bar{\delta}_{k} \phi_{n k}(z)
$$

found by interpolation to $f(z)$ at the $n$ points $a_{n k}$, that is, by the requirements:

$$
\delta_{k} f_{n}\left(a_{n k}\right)=\delta_{k} f\left(a_{n k}\right), \quad k=1,2, \cdots, n .
$$

Then the question arises: What conditions should be imposed on $a_{n k}$ in order that, for every function $f(z)$ of class $S_{\alpha}$, the corresponding sequence of functions $f_{n}(z)$ converges to $f(z)$ on $K$, uniformly on every closed point set interior to $K$ ?

In a previous paper $\left({ }^{2}\right)$, the present writer has obtained some results in the particular case where $\alpha=2$. The object of the present discussion is to generalize and, in some respects, to complete, the previous results by the following theorems:

THEOREM A. A sufficient condition is that the set $a_{n k}$ be equivalent to a set $b_{n k}$ for which $\left({ }^{3}\right)$

$$
\lim _{n \rightarrow \infty} n^{\alpha-1} \prod_{k=1}^{n}\left|b_{n k}\right|^{2}=0 .
$$

The condition is also necessary if the set $a_{n k}$ is equivalent to a set $b_{n k}$ such that, for each sufficiently large $n$, the $n$ points $b_{n k}$ are equally spaced on a circle $|z|=r_{n}, 0<r_{n}<1$.

TheOREM B. A necessary condition is that

$$
\lim _{n \rightarrow \infty} \prod_{k=1}^{n}\left|a_{n k}\right|=0 .
$$

The condition is also sufficient if $1<\alpha \leqq 2$, and if the set $a_{n k}$ is equivalent to a set $b_{n k}$ such that, for each sufficiently large $n$, the $n$ points $b_{n k}$ all lie on a diameter $d_{n}$ of the unit circle $|z|=1$.

Whether the restriction $\alpha \leqq 2$ can be removed from the last part of Theorem $B$ is a question which the present writer hopes to be able to consider on a later occasion.

(2) Yu-cheng Shen, Interpolation to certain analytic functions by rational functions, Trans. Amer. Math. Soc. vol. 60 (1946) pp. 12-21.

(3) In formulas (A) and (B), any factor which is zero should be omitted. 
As will be seen in $\S 2$, the limit class $S_{1}$ consists of all those functions $f(z)=\sum a_{n} z^{n},|z|<1$, for which the series $\sum\left|a_{n}\right|^{2}$ converges. Interpolation to functions of this class by rational functions of the form (1.3) with $\alpha=1$ has been treated by J. L. Walsh and other authors $\left({ }^{4}\right)$. This class is excluded from the following discussion.

For the sake of simplicity, we shall write $a_{k}$ for $a_{n k}$ when $n$ is fixed. Unless the contrary is explicitly stated, it is understood that $\alpha>1$ is a fixed number.

2. Functions of class $S_{\alpha}$. If $f(z)=z^{n}, n=0,1, \cdots$, then

$$
\mathfrak{R}_{\alpha}\left(z^{n} ; \rho\right)=(\alpha-1) \int_{0}^{\rho}(1-\rho)^{\alpha-2} \rho^{n} d \rho, \quad 0<\rho<1 .
$$

Since the integrand is positive, $\mathfrak{M}_{\alpha}\left(z^{n} ; \rho\right)$ increases monotonically with $\rho$, $0<\rho<1$. For an arbitrary function $f(z)=\sum_{n=0}^{\infty} a_{n} z^{n},|z|<1$, we have

$$
\mathfrak{M}_{\alpha}(f ; \rho)=\sum_{n=0}^{\infty} \mathfrak{M}_{\alpha}\left(z^{n} ; \rho\right)\left|a_{n}\right|^{2}, \quad 0<\rho<1 .
$$

It follows that $\mathfrak{M}_{\alpha}(f ; \rho)$ is nondeceasing as $\rho$ increases. Hence if $f(z)$ is of class $S_{\alpha}, \mathfrak{M}_{\alpha}(f ; \rho)$ has a limit $\mathfrak{M}_{\alpha}(f)$ as $\rho$ approaches unity. $\mathfrak{M}_{\alpha}(f)$ will be called the norm of $f(z)$ on $K$. The norms of $z^{n}$ are

$$
\begin{aligned}
& c_{0}^{(\alpha)}=\mathfrak{M}_{\alpha}(1)=1 ; \\
& c_{n}^{(\alpha)}=\mathfrak{M}_{\alpha}\left(z^{n}\right)=\frac{\Gamma(\alpha) \Gamma(n+1)}{\Gamma(\alpha+n)}=\frac{n !}{\alpha(\alpha+1) \cdots(\alpha+n-1)}, \\
& n=1,2, \cdots .
\end{aligned}
$$

For the function $f(z)$ to belong to class $S_{\alpha}$, it is necessary and sufficient that the series

$$
c_{0}^{(\alpha)}\left|a_{0}\right|^{2}+c_{1}^{(\alpha)}\left|a_{1}\right|^{2}+\cdots
$$

should converge. It is sufficient because, for $0<\rho<1$,

$$
\mathfrak{M}_{\alpha}(f ; \rho) \leqq \sum_{n=0}^{\infty} c_{n}^{(\alpha)}\left|a_{n}\right|^{2}
$$

It is necessary because, for $0<\rho<1$, and for arbitrary $N$,

$$
\sum_{n=0}^{N} \mathfrak{M}_{\alpha}\left(z^{n} ; \rho\right)\left|a_{n}\right|^{2} \leqq \mathfrak{M}_{\alpha}(f ; \rho) \leqq \mathfrak{M}_{\alpha}(f),
$$

(4) For interpolation to functions of this class and for the relevant literature, see J. L. Walsh, Interpolation and approximation by rational functions in the complex domain, Amer. Math. Soc. Colloquium Publications, vol. 20, 1935. (This book has been inaccessible to the present writer since the war.) 
and therefore

$$
\sum_{n=0}^{\infty} c_{n}^{(\alpha)}\left|a_{n}\right|^{2} \leqq \mathbb{M}_{\alpha}(f)
$$

It follows from (i) and (ii) that, if $f(z)$ is of class $S_{\alpha},(2.2)$ converges to $\mathfrak{M}_{\alpha}(f)$. Moreover, since $c_{n}^{(\alpha)}(n>0)$ decreases with $1 / \alpha, \alpha>\beta$ implies $\mathfrak{M}_{\alpha}(f)$ $\leqq \mathfrak{M}_{\beta}(f)$, where the equality sign occurs only when $f(z)$ is a constant.

If we put $\alpha=1$ in (2.2), the series becomes $\sum\left|a_{n}\right|^{2}$. Hence the limit class $S_{1}$ may be defined as consisting of functions $f(z)$ for which this latter series converges or, what amounts to the same thing, the integral

$$
\mu(f ; \rho)=\frac{1}{2 \pi} \int_{0}^{2 \pi}\left|f\left(r e^{i \theta}\right)\right|^{2} d \theta, \quad \rho=r^{2},
$$

is bounded for $0<\rho<1$. This is the well known class $L_{2}$, and will be excluded from the following discussion.

From the relation

$$
\frac{1}{(1-z \bar{t})^{\alpha}}=\sum_{n=0}^{\infty} \frac{z^{n} \bar{t}^{n}}{c_{n}^{(\alpha)}}, \quad|z|<1,|t| \leqq 1,
$$

it can be verified that the integral representation

$$
f(z)=\frac{\alpha-1}{\pi} \iint_{|z|<1} \frac{\left(1-|t|^{2}\right)^{\alpha-2} f(t)}{(1-z \bar{t})^{\alpha}} d S, \quad|z|<1,
$$

is valid for any function $f(z)$ of class $S_{\alpha}$, or, more generally, for any function $f(z)$ analytic on $K$, and such that $\left(1-|\dot{z}|^{2}\right)^{\alpha-2} f(z)$ is integrable on $K$.

We conclude the preliminary discussion by a couple of lemmas.'

LEMMA. 1. For any function $f(z)$ of class $S_{\alpha}$ which does not vanish identically, the inequality

$$
\mu(\rho) \equiv \mu(f ; \rho)<\frac{\mathfrak{M}_{\alpha}(f)}{(1-\rho)^{\alpha-1}}, \quad 0<\rho<1,
$$

is valid (where $\mu(f ; \rho)$ has the same meaning as in $(2.3)$ ).

For arbitrary $\rho$ and $\rho^{\prime}, 0<\rho<\rho^{\prime} \leqq 1$, set

$$
M\left(\rho, \rho^{\prime}\right)=(\alpha-1) \int_{\rho}^{\rho^{\prime}}(1-\rho)^{\alpha-2} \mu(\rho) d \rho .
$$

Since $f(z) \not \equiv 0$ implies $M(\rho, 1)<\mathfrak{M}_{\alpha}(f)$, it is sufficient to prove

$$
\mu(\rho) \leqq \frac{M(\rho, 1)}{(1-\rho)^{\alpha-1}}, \quad 0<\rho<1 .
$$


Subject (2.5) to the real transformation $x=1-(1-\rho)^{\alpha-1}, \rho=1$ $-(1-x)^{1 /(\alpha-1)}$ (which reduces to an identity if $\left.\alpha=2\right)$. As $\rho$ increases from 0 to $1, x$ does also. Thus (2.5) becomes

$$
M^{*}\left(x, x^{\prime}\right)=\int_{x}^{x^{\prime}} \mu^{*}(x) d x,
$$

where $x, x^{\prime}, M^{*}\left(x, x^{\prime}\right)$ and $\mu^{*}(x)$ correspond to $\rho, \rho^{\prime}, M\left(\rho, \rho^{\prime}\right)$ and $\mu(\rho)$ respectively. By the mean value theorem (which is applicable since $\mu^{*}(x)$ is a continuous function in $0 \leqq x<1)$, and by the monotonic property of $\mu^{*}(x)$, we have

$$
M^{*}\left(x, x^{\prime}\right)=\left(x^{\prime}-x\right) \mu^{*}\left(x^{\prime \prime}\right) \geqq\left(x^{\prime}-x\right) \mu^{*}(x), \quad x<x^{\prime \prime}<x^{\prime}<1,
$$

whence

$$
\mu^{*}(x) \leqq \frac{M^{*}\left(x, x^{\prime}\right)}{x^{\prime}-x} .
$$

Since, for fixed $x$, this is true for arbitrary $x^{\prime}, x<x^{\prime}<1$, we may allow $x^{\prime}$ to approach unity:

$$
\mu^{*}(x) \leqq \frac{M^{*}(x, 1)}{1-x}
$$

When this is transformed back to $\rho,(2.6)$ results. The lemma is thus proved.

LEMMA 2. Let $f(z)$ be a function of class $S_{\alpha}, \alpha>1$, which does not vanish at $z=0$, but does vanish at $a_{1}, a_{2}, \cdots, a_{n}$, where $0<\left|a_{1}\right| \leqq\left|a_{2}\right| \leqq \cdots \leqq\left|a_{n}\right|<1$. Then

$$
|f(0)|^{2}<\left(1+\frac{\alpha-1}{n}\right)^{n}\left(1+\frac{n}{\alpha-1}\right)^{\alpha-1}\left|a_{1} a_{2} \cdots a_{n}\right| 2 \mathfrak{M}_{\alpha}(f) .
$$

The proof is based on a method which is due to Montel( $\left.{ }^{5}\right)$. Let $r, 0<r<1$, be so chosen that the first $k(0<k<n)$ points $a_{1}, a_{2}, \cdots, a_{k}$ are interior to the circle $|z|=r$. Set

$$
\phi(z)=f(z) \prod_{i=1}^{k} \frac{r^{2}-\bar{a}_{i} z}{r\left(z-a_{i}\right)} .
$$

Then $\phi(z)$ is analytic for $|z|<1$, and $|\phi(z)|=|f(z)|$ on $|z|=r$. Hence

$$
|\phi(0)|^{2}<\frac{1}{2 \pi} \int_{0}^{2 \pi}\left|\phi\left(r e^{i \theta}\right)\right|^{2} d \theta=\mu(f ; \rho), \quad \rho=r^{2} .
$$

(\$) Paul Montel, Leģons sur les familles normales de fonctions analytiques et leurs applications, Paris, 1927, p. 185. 
It follows from (2.7) and Lemma 1 that

$$
\frac{\rho^{k}}{\left|a_{1} a_{2} \cdots a_{k}\right|^{2}}|f(0)|^{2}<\frac{\mathfrak{M}_{\alpha}(f)}{(1-\rho)^{\alpha-1}},
$$

that is,

$$
|f(0)|^{2}<\frac{\left|a_{1} a_{2} \cdots a_{k}\right|^{2}}{\rho^{k}(1-\rho)^{\alpha-1}} \mathfrak{M}_{\alpha}(f) .
$$

Since $\rho \leqq\left|a_{i}\right|^{2}$ for $i=k+1, k+2, \cdots, n$, we have

$$
|f(0)|^{2}<\frac{\left|a_{1} a_{2} \cdots a_{n}\right|^{2}}{\rho^{n}(1-\rho)^{\alpha-1}} \mathfrak{M}_{\alpha}(f) .
$$

An inspection of the above argument reveals that (2.8) is valid for every $\rho$, $0<\rho<1$; it is independent of the special manner in which $r$ has been chosen. Hence (2.8) holds also at any point $\rho_{0}$ at which the function $\rho^{n}(1-\rho)^{\alpha-1}$ attains its maximum in the interval $0<\rho<1$. The existence of $\rho_{0}$ follows from Rolle's Theorem, and its value is found to be unique and equal to $n /(n+\alpha-1)$. When this value is substituted in the right-hand side of $(2.8)$, the result is the inequality asserted by Lemma 2 . The proof is complete.

3 . The remainder $f(z)-f_{n}(z)$. In the case where the $n$ points $a_{1}, a_{2}, \cdots, a_{n}$ are distinct, the determinant $\Delta_{n}$ of the system of equations (1.4) is equal to

$$
D_{n} \equiv D_{n}\left(a_{1}, a_{2}, \cdots, a_{n} ; \bar{a}_{1}, \bar{a}_{2}, \cdots, \bar{a}_{n}\right)=\left|\frac{1}{\left(1-a_{i} \bar{a}_{j}\right)^{\alpha}}\right| .
$$

Since, by (2.4),

$$
\frac{\alpha-1}{\pi} \iint_{|t|<1} \frac{\left(1-|t|^{2}\right)^{\alpha-2}}{\left(1-a_{i} \bar{t}\right)^{\alpha}\left(1-\bar{a}_{j} t\right)^{\alpha}} d S=\frac{1}{\left(1-a_{i} \bar{a}_{j}\right)^{\alpha}},
$$

it follows that $\Delta_{n}$ is the Gramian determinant $\left({ }^{\circ}\right)$ for the $n$ linearly independent functions

$$
\frac{(\alpha-1)^{1 / 2}\left(1-|z|^{2}\right)^{(\alpha-2) / 2}}{\pi^{1 / 2}\left(1-\bar{a}_{i} z\right)^{\alpha}}, \quad i=1,2, \cdots, n .
$$

Hence $\Delta_{n}>0$, and the function $f_{n}(z)$ is uniquely determined for each $n$. The remainder $f(z)-f_{n}(z)=R_{n}(z)$ is given by

$$
R_{n}(z)=\frac{\alpha-1}{\pi} \iint_{|t|<1}\left(1-|t|^{2}\right)^{\alpha-2} f(t) r_{n}(z ; t) d S, \quad|z|<1,
$$

where

(') See Gerhard Kowalewski, Einführung in die determinantentheorie, 2d ed., Berlin and Leipzig, p. 224. 


$$
\begin{aligned}
r_{n}(z ; \bar{t}) & =\frac{\Delta_{n+1}(z ; \bar{t})}{\Delta_{n}}, \\
\Delta_{n+1}(z ; \bar{t}) & =D_{n+1}\left(a_{1}, a_{2}, \cdots, a_{n}, z ; \bar{a}_{1}, \bar{a}_{2}, \cdots, \bar{a}_{n}, \bar{t}\right) .
\end{aligned}
$$

The verification is immediate; the right-hand side of (3.2) is $f(z)$ minus a function of the form (1.3) and vanishes at $z=a_{1}, a_{2}, \cdots, a_{n}$. In a similar manner, it can be verified that, even if the $n$ points $a_{1}, a_{2}, \cdots, a_{n}$ are not distinct, (3.2) is still true, provided that $\Delta_{n}$ is interpreted as implying

$$
\Delta_{n}=\delta_{n} \delta_{n-1} \cdots \delta_{1} \overline{\delta_{n} \delta_{n-1} \cdots \delta_{1}} D_{n}\left(a_{1}, a_{2}, \cdots, a_{n} ; \bar{a}_{1}, \bar{a}_{2}, \cdots, \bar{a}_{n}\right)
$$

and $\Delta_{n+1}(z ; \bar{t})$ is interpreted in a similar manner.

On comparing (3.2) with a well known formula in the theory of orthogonal functions $\left({ }^{7}\right)$, we see that $f_{n}(z)$ is also the unique function of the form (1.3) for which the norm $\mathfrak{M}_{\alpha}\left(f-f_{n}\right)$ on $K$ is least. Thus our problem of interpolation to $f(z)$ by $f_{n}(z)$ is equivalent to one of approximation to $f(z)$ by $f_{n}(z)$ in the sense of least squares-measured by surface integrals on $K$ with respect to the weight function $(\alpha-1) \pi^{-1}\left(1-|z|^{2}\right)^{\alpha-2}$.

In particular, if $f(z)=(1-\zeta z)$, where $\zeta,|\zeta|<1$, is a parameter, the corresponding $R_{n}(z)$ is precisely $r_{n}(z ; \xi)$. Hence $r_{n}(z ; \xi)$ is the unique function of the form

$$
\frac{1}{(1-\zeta z)^{\alpha}}+\sum_{k=1}^{n} A_{n k} \bar{\delta}_{k} \frac{1}{\left(1-\bar{a}_{n k} z\right)^{\alpha}}
$$

whose norm on $K$ is least. This norm is given by

$$
\mathfrak{M}_{\alpha}\left(r_{n}(z ; \xi)\right)=r_{n}(\zeta ; \xi),
$$

as can be verified by multiplying $\pi^{-1}(\alpha-1)\left(1-|z|^{2}\right)^{\alpha-2} r_{n}(z ; \xi)$ by the conjugate of (3.4) and integrating. Furthermore, $\left.r_{n}(\zeta ; \bar{\zeta}) \leqq 1-|\zeta|^{2}\right)^{-\alpha}$.

Now, applying Schwarz's inequality to (3.2) and using (3.5), we have

$$
\left|R_{n}(z)\right|^{2} \leqq \mathfrak{M}_{\alpha}(f) \cdot r_{n}(z ; \bar{z}) \leqq \frac{\mathfrak{M}_{\alpha}(f)}{\left(1-|z|^{2}\right)^{\alpha}} \quad|z|<1 .
$$

Thus the study of $R_{n}(z)$ is reduced to a study of $r_{n}(z ; \bar{z})$.

4. The invariant form of $r_{n}(z ; \bar{t})$. The determinant $D_{n}$ in (3.1) is analytic in the $2 n$ independent variables $a_{i}, \bar{a}_{i}$ in the region $R:\left|a_{i}\right|<1,\left|\bar{a}_{i}\right|<1$, $i=1,2, \cdots, n$. As a function in $a_{1}$, it vanishes at $a_{1}=a_{2}, a_{3}, \cdots, a_{n}$. Hence

$$
D_{n}=F_{1} \cdot \prod_{i=2}^{n}\left(a_{1}-a_{i}\right),
$$

where $F_{1}$ is analytic in $R$. Continuing the process, we arrive at

(7) Gerhard Kowalewski, ibid. pp. 227-229. 


$$
D_{n}=F_{2 n-2} P_{n} \bar{P}_{n}, \quad P_{n}=\prod_{i<j}\left(a_{i}-a_{j}\right),
$$

where $F_{2 n-2}$ is analytic in $R$. From this we deduce

$$
\Delta_{n}=F_{2 n-2}\left|\delta_{n} \delta_{n-1} \cdots \delta_{1} P_{n}\right|^{2},
$$

and similarly

$$
\Delta_{n+1}(z ; \bar{t})=\Phi_{2 n}(z ; \bar{t})\left|\delta_{n} \delta_{n-1} \cdots \delta_{1} P_{n}\right|^{2} \prod_{i=1}^{n}\left(\frac{z-a_{i}}{1-\bar{a}_{i} z}\right)\left(\overline{\frac{t-a_{i}}{1-\bar{a}_{i} t}}\right) .
$$

Thus $r_{n}(z ; \bar{t})$ can be written in the form

$$
r_{n}(z ; \bar{t})=A_{n}(z ; \bar{t}) B_{n}(z) \overline{B_{n}(t)},
$$

where

$$
A_{n}(z ; \bar{t})=\frac{\Phi_{2 n}(z ; \bar{t})}{F_{2 n-2}}, \quad B_{n}(z)=\prod_{i=1}^{n} \frac{z-a_{i}}{1-\bar{a}_{i} z} .
$$

Since the symbol $\delta$ does not appear in the right-hand side of (4.1), this formula has the advantage that it is not affected by the coincidence of some or all of the $n$ points $a_{i}$. Another advantage of (4.1) is its invariant property under (1.2), which can be conveniently stated as follows:

LEMMA 3. If the $n+2$ points $a_{1}, a_{2}, \cdots, a_{n}, z, t$ on $K$ are transformed by (1. 2) into $b_{1}, b_{2}, \cdots, b_{n}, \zeta, \tau$, then

$$
(1-z \bar{t})^{\alpha} r_{n}(a, z ; \bar{a}, \bar{t})=(1-\zeta \bar{\tau})^{\alpha} r_{n}(b, \zeta ; \bar{b}, \bar{\tau})
$$

(where we have introduced new notation for $r_{n}(z ; t)$ to indicate to which set of points it corresponds).

This can be verified as follows. In the first place, $B_{n}(z)$ is invariant under (1.2). Secondly, when the $a$ 's are distinct, and the $a$ 's and the $b$ 's are different from zero, the invariance of $(1-z \bar{t})^{\alpha} r_{n}(z ; \bar{t})$ follows from $(3.3)$ and the relation

$$
1-b_{i} \bar{b}_{j}=\frac{1-|c|^{2}}{\left(1-\bar{c} a_{i}\right)\left(1-c \bar{a}_{i}\right)}\left(1-a_{i} \bar{a}_{j}\right) .
$$

Hence, when the $a$ 's and the $b$ 's are restricted as above, the function

$$
(1-z \bar{t})^{\alpha} A_{n}(z ; \bar{t})=\frac{(1-z \bar{t})^{\alpha} r_{n}(z ; \bar{t})}{B_{n}(z) \overline{B_{n}(t)}}=\frac{(1-z \bar{t})^{\alpha} \Phi_{2 n}(z ; \bar{t})}{F_{2 n-2}}
$$

is invariant under (1.2). Since the forms of $F_{2 n-2}$ and $\Phi_{2 n}(z ; \bar{t})$ are not affected by the removal of the above restriction, the invariance of $(1-z \bar{t})^{\alpha} A_{n}(z ; \bar{t})$ subsists in the general case. This can be justified by a limit process. Lemma 3 is thus verified. 
LeMmA 4. For any $n+1$ fixed points $a_{1}, a_{2}, \cdots, a_{n}$ and $t$ on $K$, the corresponding function $A_{n}(z ; \bar{t})$ satisfies the inequality:

$$
A_{n}(t ; \bar{t})<\frac{M_{n}^{\alpha-1}}{\left(1-|t|^{2}\right)^{\alpha}}, \quad M=\left(\frac{\alpha e}{\alpha-1}\right)^{\alpha-1}, \quad \alpha>1 .
$$

In virtue of the invariant property of $\left(1-|z|^{2}\right)^{\alpha} A_{n}(z ; \bar{z})$, it is sufficient to consider the case $t=0$. If the $a$ 's are different from zero, then, by applying Lemma 2 to the function $\phi(z)=A_{n}(z ; 0) B_{n}(z)$, and by observing that

$$
\mathfrak{M}_{\alpha}(\phi)=A_{n}(0 ; 0),
$$

we have

$$
A_{n}(0 ; 0)<\left(1+\frac{\alpha-1}{n}\right)^{n}\left(1+\frac{n}{\alpha-1}\right)^{\alpha-1}
$$

The case where some of the $a$ 's are zero can be readily disposed of by limit process. In each case, we have

$$
A_{n}(0 ; 0)<\left(\frac{\alpha e}{\alpha-1}\right)^{\alpha-1} n^{\alpha-1}
$$

because $n \geqq 1$ implies

$$
\left(1+\frac{\alpha-1}{n}\right)^{n}<e^{\alpha-1}, \quad 1+\frac{n}{\alpha-1} \leqq \frac{\alpha n}{\alpha-1} .
$$

The proof is complete.

5. Proof of the first part of Theorem A. We are now in a position to prove the first part of Theorem A. Let $f(z)$ be an arbitrary function of class $S_{\alpha}$, and $R_{n}(z)$ the corresponding remainder. Then, by (3.6)

$$
\left|R_{n}(z)\right|^{2} \leqq \frac{\mathfrak{M}_{\alpha}(f)}{\left(1-r^{2}\right)^{\alpha}}, \quad|z| \leqq r<1 .
$$

It follows that the functions $R_{n}(z)$ form a normal family on $K$. From every subsequence of $R_{n}(z)$ can be extracted a subsequence $R_{n_{i}}(z)$ which converges to an analytic function $R(z)$ on $K$, uniformly on any closed point set on $K$. To prove the first part of Theorem $A$, it is sufficient to prove that, under condition (A), every such limit function $R(z)$ is identically zero. For simplicity, we shall take $R_{n}(z)$ for $R_{n_{i}}(z)$.

Let $b_{n k}$ be the set of points which satisfies condition (A) and into which the set $a_{n k}$ is transformed by (1.2). Let $R^{*}(\zeta)$ and $R_{n}^{*}(\zeta)$ be the transforms of $R(z)$ and $R_{n}(z)$ respectively. By (3.6), we have

$$
\left|R_{n}(z)\right|^{2} \leqq \mathfrak{M}_{\alpha}(f) r_{n}(a, z ; \bar{a}, \bar{z}),
$$

where we have changed the notation to that in Lemma 3. But, by Lemma 3 
and Lemma 4,

$$
\begin{aligned}
& r_{n}(a, z ; \bar{a}, \bar{z})=\left(\frac{1-|\zeta|^{2}}{1-|z|^{2}}\right)^{\alpha} r_{n}(b, \zeta ; \bar{b}, \bar{\zeta}) \\
& \leqq \frac{M}{\left(1-|z|^{2}\right)^{\alpha}} n^{\alpha-1}\left|B_{n}(\zeta)\right|^{2}, \quad|\zeta|<1 \text {, }
\end{aligned}
$$

and, by (1.2),

$$
\frac{1}{1-|z|^{2}}=\frac{|\lambda+\bar{c} \zeta|^{2}}{\left(1-|c|^{2}\right)\left(1-|\zeta|^{2}\right)}<\frac{1+|c|}{1-|c|} \frac{1}{1-|\zeta|^{2}}, \quad|\zeta|<1 .
$$

Therefore

$$
\left|R_{n}^{*}(\zeta)\right|^{2} \leqq\left(\frac{1+|c|}{1-|c|}\right)^{\alpha} \frac{M \mathfrak{M}_{\alpha}(f)}{\left(1-|\zeta|^{2}\right)^{\alpha}} n^{\alpha-1}\left|B_{n}(\zeta)\right|^{2}, \quad|\zeta|<1 .
$$

Now suppose that $R(z) \not \equiv 0$. Then $R^{*}(\zeta) \not \equiv 0$, and consequently there exists an $r, 0<r<1$, such that $\left|R^{*}(\zeta)\right|$ has a positive minimum $m$ on the circle $|\zeta|=r$. Hence, for $n$ sufficiently large, $\left|R_{n}^{*}(\zeta)\right|>m / 2$ on $|\zeta|=r$. In order to arrive at a contradiction, three cases are to be distinguished.

First, suppose that there exists an $n_{0}$ such that $n>n_{0}$ implies $\left|b_{n k}\right|>r$. Then, since $B_{n}(\zeta), n>n_{0}$, does not vanish in $|\zeta| \leqq r,\left|B_{n}(\zeta)\right|$ attains its minimum in $|\zeta| \leqq r$ at a point $\zeta_{n}$ on the circle $|\zeta|=r$ :

$$
\left|B_{n}\left(\zeta_{n}\right)\right|<\left|B_{n}(0)\right|=\left|b_{n 1} b_{n 2} \cdots b_{n n}\right| \text {. }
$$

Hence, at $\zeta=\zeta_{n}$, (5.1) becomes

$$
\left|R_{n}^{*}\left(\zeta_{n}\right)\right|^{2}<\left(\frac{1+|c|}{1-|c|}\right)^{\alpha} \frac{M \mathfrak{M}_{\alpha}(f)}{\left(1-r^{2}\right)^{\alpha}} n^{\alpha-1} \prod_{k=1}^{n}\left|b_{n k}\right|^{2} .
$$

The right-hand side, by hypothesis, approaches zero with $1 / n$. It follows that, for $n$ sufficiently large, $\left|R_{n}^{*}\left(\zeta_{n}\right)\right|<m / 2$. This is a contradiction.

Secondly, suppose that there exists $k_{0}$ such that $k>k_{0}$ implies $\left|b_{n k}\right|>r$. In this case, we simply omit the first $k_{0}$ factors $\left(\zeta-b_{n k}\right) /\left(1-\zeta b_{n k}\right), k=1$, $2, \cdots, k_{0}$, from $B_{n}(\zeta)$, and repeat the above argument. This leads to the inequality

$$
\left|R_{n}^{*}\left(\zeta_{n}\right)\right|^{2}<\left(\frac{1+|c|}{1-|c|}\right)^{\alpha} \frac{M \mathfrak{M}_{\alpha}(f)}{\left(1-r^{2}\right)^{\alpha}} n^{\alpha-1} \prod_{k=k_{0}+1}^{n}\left|b_{n k}\right|^{2}
$$

which differs from (5.2) only by the product $\left|b_{n 1} b_{n 2} \cdots b_{n k_{0}}\right|^{2}$. This product, when it appears in condition (A), can not be zero, because, by definition, any zero factor should be omitted; nor can it approach zero, because the points $b_{n k}$ have no limit point interior to the unit circle. Hence condition (A) im- 
plies that the right-hand side of (5.3) approaches zero with $1 / n$, and we arrive at a contradiction as before.

Finally, there remains the (trivial) case where $k_{1}$ exists so that, for $n$ sufficiently large, at most $k_{1}$ points $b_{n k}$ are exterior to $|\zeta|=r$. Since $\left|b_{n k}\right| \leqq r$ implies

$$
\left|\frac{\zeta-b_{n k}}{1-\zeta b_{n k}}\right| \leqq \frac{|\zeta|+r}{1+r|\zeta|}
$$

it follows that, for $|\zeta|=r$, the right-hand side of (5.1) is of an order not higher than

$$
n^{\alpha-1}\left(\frac{2 r}{1+r^{2}}\right)^{2 n-2 k_{1}}
$$

But this quantity approaches zero with $1 / n$, and again we are led to a contradiction. The proof is complete.

6. Computation of $r_{n}(0 ; 0)$. To prepare for the proof of the remaining part of Theorem A, let us compute the value of $r_{n}(0 ; 0)$ for the set

$$
b_{k}=r x_{k}, \quad x_{k}=e^{i 2 k \pi / n},
$$$$
k=1,2, \cdots, n \text {. }
$$

For this purpose, the so-called cyclic determinant can be used to advantage. A cyclic determinant is one of the form $\left(^{8}\right)$

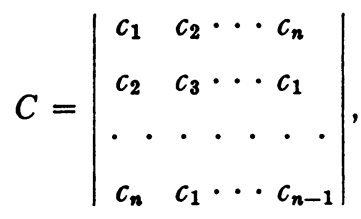

whose value is given by

$$
C=(-1)^{(n-1)(n-2) / 2} f\left(x_{1}\right) f\left(x_{2}\right) \cdots f\left(x_{n}\right),
$$

where

$$
f(x)=c_{1}+c_{2} x+c_{3} x^{2}+\cdots+c_{n} x^{n-1},
$$

$x_{1}, x_{2}, \cdots, x_{n}$ having the same meaning as in (6.1). Since we have to make some alterations in (6.2), it is appropriate to indicate the proof of (6.3). Set

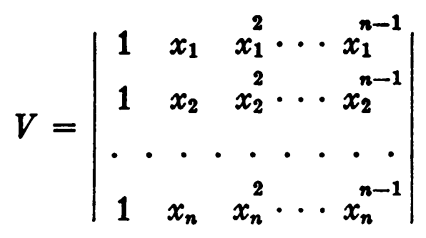

(8) Gerhard Kowalewski, ibid. pp. 105-107. See the literature at the end of the book, p. 300. 
and perform the multiplication $C V$ row by row. The element $e_{h k}$ in the $h$ th row and the $k$ th column of $C V$ can be reduced to the form $x_{k}^{n-n+1} f\left(x_{k}\right)$. This reduction depends on the periodic property of the $x$ 's only; it does not involve any transformation of the matrix of $C V$. Thus

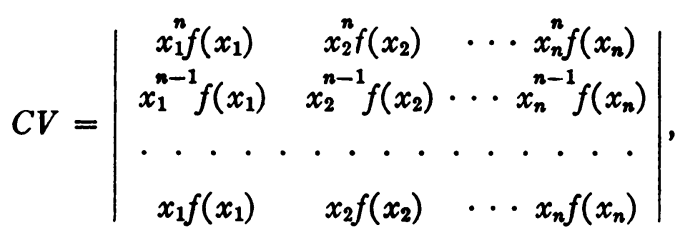

which, when simplified, gives (6.3).

Now, if we replace the last row of $C$ by $1,1, \ldots, 1$, and denote by $C^{*}$ the determinant thus obtained, the product $C^{*} V$ is also of the form (6.4) except that the last row becomes $0,0, \cdots, 0, n$. Since $x_{n}=1$, and $\left(x_{1} x_{2} \cdots x_{n}\right)^{2}$ $=1$, we have

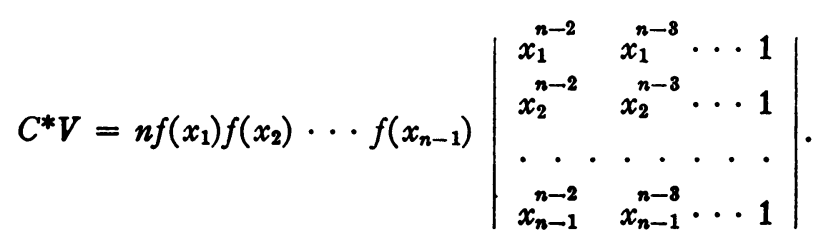

When $V$ is divided by the determinant on the right-hand side, the result is found to be $\left(^{9}\right)$

$$
(-1)^{(n-1)(n-2) / 2}\left(1-x_{1}\right)\left(1-x_{2}\right) \cdots\left(1-x_{n-1}\right)=(-1)^{(n-1)(n-2) / 2} n .
$$

Hence,

$$
C^{*}=(-1)^{(n-1)(n-2) / 2} f\left(x_{1}\right) f\left(x_{2}\right) \cdots f\left(x_{n-1}\right),
$$

and, if $C \neq 0$,

$$
\frac{C^{*}}{C}=\frac{1}{f(1)}
$$

Now proceed to compute $\Delta_{n}=D_{n}\left(b_{1}, b_{2}, \cdots, b_{n} ; b_{1}, b_{2}, \cdots, b_{n}\right)$ (cf. $\S 3$ ). Since $b_{h} \bar{b}_{k}=r^{2} x_{h} \bar{x}_{k}=\rho x_{1}^{n+h-k}, \rho=x^{2}$, we have

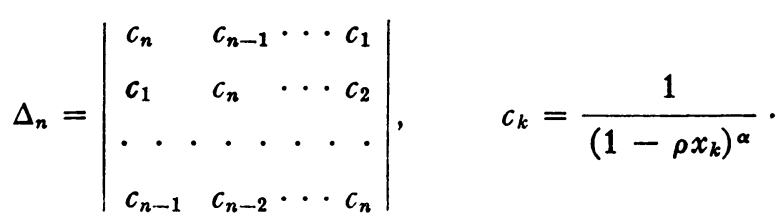

(9) For the various expansions of $V$, see Gerhard Kowalewski, ibid. pp. 39-40. 
Let $\Delta_{n h}$ denote the determinant obtained from $\Delta_{n}$ by replacing the $h$ th row by $1,1, \cdots, 1$. We note that

$$
\Delta_{n 1}=\Delta_{n 2}=\cdots=\Delta_{n n} .
$$

For, if (when $h>1$ ) we shift the rows of $\Delta_{n h}$ so that the first, second, $\cdots$, $(h-1)$ th rows becomes the $n$ th, $(n-1)$ th, $\cdots,(n-h+2)$ th rows respectively, and then shift the columns in the same manner, $\Delta_{n h}$ becomes $\Delta_{n 1}$. On the other hand, if we shift the $h$ th row of $\Delta_{n h}$ to the $n$th row, the result is $(-1)^{n-h} \Delta_{n 1}$

With these observations, we can readily expand $\Delta_{n+1}(0 ; 0)$ according to the last column:

$$
\Delta_{n+1}(0 ; 0)=\Delta_{n}+\sum_{h=1}^{n}(-1)^{n+1+h}(-1)^{n-h} \Delta_{n 1}=\Delta_{n}-n \Delta_{n 1} .
$$

Hence

$$
r_{n}(0 ; 0)=1-\frac{n \Delta_{n 1}}{\Delta_{n}}=1-\frac{n \Delta_{n n}}{\Delta_{n}} .
$$

Since $\Delta_{n}$ differs from $C$ in the same way as $\Delta_{n n}$ from $C^{*}$, we conclude that

$$
r_{n}(0 ; 0)=1-\frac{n}{f(1)},
$$

where

$$
\frac{f(1)}{n}=\frac{1}{n} \sum_{h=1}^{n} \frac{1}{\left(1-\rho x_{h}\right)^{\alpha}}=1+\sum_{k=1}^{\infty} \frac{\alpha(\alpha+1) \cdots(\alpha+k n-1)}{(k n) !} \rho^{k n} .
$$

This is the value of $r_{n}(0 ; 0)$ desired.

7. Proof of the second part of Theorem A. Let the given function be $(1-\bar{c} z)^{-\alpha}$, where $c$ is the constant in (1.2). At $z=c$, the corresponding remainder is

$$
r_{n}(a, c ; \bar{a}, \bar{c})=\frac{r_{n}(b, 0 ; \bar{b}, 0)}{\left(1-|c|^{2}\right)^{\alpha}}
$$

Since $r_{n}(b, 0 ; b, 0)$ is invariant under rotation, we may assume that the $n$th point $b_{n n}$ is real and positive. Then the value of $r_{n}(b, 0 ; b, 0)$ is given by (6.5) with $\rho=\rho_{n}$. In order that the remainder approaches zero with $1 / n$, it is necessary that

$$
\lim _{n \rightarrow \infty} \frac{\alpha(\alpha+1) \cdots(\alpha+n-1)}{n !} \rho_{n}^{n}=0 .
$$

To prove the second part of Theorem A, we need merely to prove the equiva- 
lence of (7.1) and (A), or, what amounts to the same thing, to prove that

$$
\log \frac{\alpha(\alpha+1) \cdots(\alpha+n-1)}{n !}-(\alpha-1) \log n
$$

remains finite as $n$ increases. Since

$$
\frac{\alpha(\alpha+1) \cdots(\alpha+n-1)}{1 \cdot 2 \cdots n}=\left(1+\frac{\alpha-1}{1}\right)\left(1+\frac{\alpha-1}{2}\right) \cdots\left(1+\frac{\alpha-1}{n}\right),
$$

and since

$$
h>\log (1+h)>h-\frac{h^{2}}{2}, \quad 0<h<1,
$$

(7.2) lies between the two numbers

$$
(\alpha-1)\left[\sum_{k=1}^{n} \frac{1}{k}-\log n\right]
$$

and

$$
(\alpha-1)\left[\sum_{k=1}^{n} \frac{1}{k}-\log n\right]-\frac{(\alpha-1)^{2}}{2} \sum_{k=1}^{n} \frac{1}{k^{2}} .
$$

Both of these approach finite limits. The proof is complete.

8. The case of simple sequence $a_{n}$. In the case where $a_{n k}=a_{k}$ for all $n$ and $k$, we state a theorem which is an obvious extention of a theorem given by Walsh $\left({ }^{10}\right)$ in connection with interpolation to functions of class $L_{2}$. The proof is omitted.

For an arbitrary set $a_{n}$ preassigned on $K$, and for a given function $f(z)$ of class $S_{\alpha}, \alpha>1$, the corresponding sequence $f_{n}(z)$ converges on $K$, uniformly on any closed point set on $K$, to a function $g(z)$, which is characterized by the fact that, among all functions of class $S_{\alpha}$ which coincide with $f(z)$ at the points $a_{n}$, $g(z)$ is the unique one whose norm on $K$ is the least. In particular, we have:

The function $\phi_{n}^{(\alpha)}(z)$ defined by $\left.{ }^{11}\right)$

$$
\phi_{n}^{(\alpha)}(z)=\frac{r_{n}^{(\alpha)}(z ; \bar{t})}{r_{n}^{(\alpha)}(t ; \bar{t})}=\frac{A_{n}^{(\alpha)}(z ; \bar{t}) B_{n}(z)}{A_{n}^{(\alpha)}(t ; \bar{t}) B_{n}(t)}
$$

is the unique function of class $S_{\alpha}$ of least norm on $K$ which vanishes at $a_{1}, a_{2}, \cdots, a_{n}$ and takes on the value unity at $z=t$.

In the last statement, it is tacitly assumed that $t$ is distinct from the $a$ 's.

(10) See the book mentioned in footnote $4, \$ 10.7$.

(II) Since we are going to consider how $r_{n}(z ; \bar{z})$ varies with $\alpha$, superscripts are introduced to indicate to which value of $\alpha$ the function $r_{n}(z ; \bar{z})$ corresponds. 
If $t$ coincides with $k$ of these points, the factor $B_{n}(t)$ in (8.1) should be replaced by $B_{n}^{(k)}(t)$, the $k$ th derivative of $B_{n}(z)$ at the point $z=t$. With this understanding, we proceed to establish the following lemma.

LEMMA 5. Let $n+1$ points $a_{1}, a_{2}, \cdots, a_{n}, t$ be preassigned on $K$. Then (1) for fixed $\alpha, \alpha>1, A_{n}^{(\alpha)}(t ; \bar{t})$ increases monotonically with $n$; and (2) for fixed $n$, $A_{n}^{(\alpha)}(t ; \bar{t})$ increases monotonically with $\alpha, \alpha>1$.

To prove (1), let $\phi_{n}^{(\alpha)}(z)$ and $\phi_{n-1}^{(\alpha)}(z)$ be defined as in (8.1), and let

$$
\psi_{n}(z)=\phi_{n-1}^{(\alpha)}(z) \frac{z-a_{n}}{1-\bar{a}_{n} z} \frac{1-\bar{a}_{n} t}{t-a_{n}} .
$$

Then $\phi_{n}^{(\alpha)}(z)$ and $\psi_{n}(z)$ both vanish at $a_{1}, a_{2}, \cdots, a_{n}$ and both take on the value unity at $z=t$. Further, we have

$$
\begin{aligned}
\mathfrak{M}_{\alpha}\left(\phi_{n}^{(\alpha)}\right) & =\frac{1}{A_{n}^{(\alpha)}(t ; \bar{t})\left|B_{n}(t)\right|^{2}}, \\
\mathfrak{M}_{\alpha}\left(\psi_{n}\right)<\left|\frac{1-\bar{a}_{n} t}{t-a_{n}}\right|^{2} \mathfrak{M}_{\alpha}\left(\phi_{n-1}^{(\alpha)}\right) & =\frac{1}{A_{n-1}^{(\alpha)}(t ; \bar{t})\left|B_{n}(t)\right|^{2}} .
\end{aligned}
$$

Statement (1) then follows from the minimum property of $\phi_{n}^{(\alpha)}(z ; \bar{t})$.

To prove (2), let $\beta>\alpha>1$, and define $\phi_{n}^{(\alpha)}(z)$ and $\phi_{n}^{(\beta)}(z)$ as above. Then both of these functions belong to class $S_{\beta}$, and both vanish at $a_{1}, a_{2}, \cdots, a_{n}$ and equal to unity at $z=t$. Because of the minimum property of $\phi_{n}^{(\beta)}(z)$, we have

$$
\mathfrak{M}_{\beta}\left(\phi_{n}^{(\beta)}\right) \leqq \mathfrak{M}_{\beta}\left(\phi_{n}^{(\alpha)}\right) \leqq \mathfrak{M}_{\alpha}\left(\phi_{n}^{(\alpha)}\right) .
$$

The first equality sign occurs when $a_{1}=a_{2}=\cdots=a_{n}=t=0$, for then $\phi_{n}^{(\alpha)}(z) \equiv \phi_{n}^{(\beta)}(z)=z^{n} / n !$. But the second equality sign cannot occur, because $\phi_{n}^{(\alpha)}\left({ }^{\alpha} z\right)$ is not a constant (cf. §2). Hence

$$
\mathfrak{M}_{\beta}\left(\phi_{n}^{(\beta)}\right)<\mathfrak{M}_{\alpha}\left(\phi_{n}^{(\alpha)}\right),
$$

which implies

$$
A_{n}^{(\alpha)}(t ; \bar{t})<A_{n}^{(\beta)}(t ; \bar{t}) .
$$

This completes the proof of Lemma 5 .

9. Proof of Theorem B. We begin with the remark: If one of the two equivalent sets $a_{n k}$ and $b_{n k}$ satisfies condition (B), so does the other. For, (1.2) gives

$$
1-|\zeta|^{2}=\frac{\left(1-|z|^{2}\right)\left(1-|c|^{2}\right)}{|1-\bar{c} z|^{2}}, \quad|z|<1,|c|<1 .
$$

Since 


$$
\frac{1-|c|}{1+|c|}<\frac{1-|c|^{2}}{|1-\bar{c} z|^{2}}<\frac{1+|c|}{1-|c|}
$$

we have

$$
\frac{1-|c|}{1+|c|}\left(1-|z|^{2}\right)<1-|\zeta|^{2}<\frac{1+|c|}{1-|c|}\left(1-|z|^{2}\right) .
$$

Hence the divergence of $\prod_{k-1}^{n}\left|a_{n k}\right|^{2}$ implies the divergence of $\prod_{k-1}^{n}\left|b_{n k}\right|^{2}$, and conversely.

To show that condition (B) is necessary for every set $a_{n k}$, we may assume that $a_{n k} \neq 0$. For, the general case can be brought into this case by a transformation of the form (1.2), and it follows from the above remark that, if (B) is necessary for the new points, it is also necessary for the original points.

Choose $f(z) \equiv 1$. Then the corresponding remainder is $r_{n}^{(\alpha)}(z ; 0)$. At $z=0$, we have, by Lemma 5, (1),

$$
r_{n}^{(\alpha)}(0 ; 0)=\left|B_{n}(0)\right|^{2} A_{n}^{(\alpha)}(0 ; 0)>\left|B_{n}(0)\right|^{2} A_{1}^{(\alpha)}(0 ; 0) .
$$

Since, by direct computation,

$$
A_{1}^{(\alpha)}(0 ; 0)=\frac{1-\left(1-\left|a_{n 1}\right|^{2}\right)^{\alpha}}{\left|a_{n 1}\right|^{2}}>1
$$

the necessity of condition (B) follows.

Turn to the sufficiency part of Theorem B. For $\alpha=2$, this is true( $\left.{ }^{12}\right)$. An immediate consequence is that $r_{n}^{(2)}(z ; \bar{z})$ converges to zero at every point $z$, $|z|<1$. For, with fixed $z, r_{n}^{(2)}(\zeta ; \bar{z})$ is the remainder corresponding to the function $1 /(1-\zeta \bar{z})^{2}$. Since this remainder converges to zero for $|\zeta|<1$, it converges to zero at $\zeta=z$.

Now let $1<\alpha<2$. In view of (3.6), and in view of the fact that the functions $R_{n}(z)$ form a normal family interior to the unit circle, it is sufficient to prove that $r_{n}^{(\alpha)}(z ; \bar{z})$ converges to zero for every fixed $z,|z|<1$. By Lemma 5 , (2), we have

$$
A_{n}^{(\alpha)}(z ; \bar{z})<A_{n}^{(2)}(z ; \bar{z}), \quad 1<\alpha<2,|z|<1 .
$$

Therefore

$$
r_{n}^{(\alpha)}(z ; \bar{z}) \leqq r_{n}^{(2)}(z ; \bar{z}), \quad 1<\alpha<2,|z|<1 .
$$

Hence, at every fixed point $z,|z|<1, r_{n}^{(\alpha)}(z ; \bar{z})$ converges to zero uniformly for $1<\alpha \leqq 2$. The proof is complete.

10. Addendum. In what follows, we shall remove the restriction $\alpha \leqq 2$ from Theorem $B$.

(19) This is proved in the paper referred to in footnote 2. 
Let $\Gamma$ denote the circle $r=\cos \theta$ in the $z$-plane, $z=r e^{i \theta}$. Then we have

$$
|z|^{2}+|1-z|^{2}=1 \text {, }
$$

$z$ on $\Gamma$.

Hence

$$
\frac{|1-z|^{2}}{1-|z|}=1+|z|<2,
$$

Let $z_{0}$ be any point interior to $\Gamma$, and let $z$ be one of the points at which $\Gamma$ intersects the circle $|z|=\left|z_{0}\right|$. Then $1-\left|z_{0}\right|=1-\left|z_{1}\right|,\left|1-z_{0}\right|<\left|1-z_{1}\right|$, and we have

$$
\frac{|1-z|^{2}}{1-|z|}<2, \quad z \text { interior to } \Gamma \text {. }
$$

LEMMA 6. Let $f(z)$ be a function which is analytic for $|z|<1$, satisfies the condition

$$
|f(z)|<\frac{M}{(1-|z|)^{\sigma}}, \quad|z|<1
$$

(where $M>0, \sigma>0$ are constants), and vanishes at the points $a_{1}, a_{2}, \cdots, a_{n}$ interior to $\Gamma$. Then, for $z$ interior to $\Gamma$, we have

$$
|f(z)| \leqq \frac{2 \sigma M}{(1-|z|)^{2 \sigma}} \prod_{k=1}^{n}\left|\frac{w-w_{k}}{1-\bar{w}_{k} w}\right|,
$$

where

$$
\begin{aligned}
w & =2 z-1, \\
w_{k} & =2 a_{k}-1,
\end{aligned}
$$$$
k=1,2, \cdots, n \text {. }
$$

From (10.1) and (10.2), we have, for $z$ interior to $\Gamma$,

$$
\left|(1-z)^{2 \sigma} f(z)\right|<\frac{|1-z|^{2 \sigma}}{(1-|z|)^{\sigma}} M<2^{\sigma} M .
$$

The function on the left-hand side, when suitably defined, is analytic interior to $\Gamma$.

Now transform the $z$-plane by (10.4), by which $\Gamma:|z-1 / 2|=1 / 2$, or $r=\cos \theta$, goes into the circle $|w|=1$ so that their centers correspond to each other. Let $F(w)$ denote the transform of $f(z)$. Then

$$
\left|\left(\frac{1-w}{2}\right)^{2 \sigma} F(w)\right|<2 \sigma M, \quad|w|<1 .
$$

The function on the left-hand side is analytic and bounded for $|w|<1$. Since this function vanishes at the points $w_{k}$ given by $(10.5)$, an application of the 
generalized form of Schwarz's lemma gives

$$
\left|\left(\frac{1-w}{2}\right)^{2 \sigma} F(w)\right| \leqq 2^{\sigma} M \prod_{k=1}^{n}\left|\frac{w-w_{k}}{1-\bar{w}_{k} w}\right|, \quad|w|<1 .
$$

When the left-hand side is transformed back to $z$, inequality (10.3) follows. The proof is complete.

Remark. Let $\Gamma(\theta)$ denote the circle which can be brought into coincidence with $\Gamma$ by a rotation $z^{\prime}=z e^{-i \theta}, 0<\theta<2 \pi$. Then Lemma 6 remains valid if we replace $\Gamma$ by $\Gamma(\theta)$ and replace (10.4) and (10.5) respectively by

$$
w=2 z e^{-i \theta}-1, \quad \quad w_{k}=2 a_{k} e^{-i \theta}-1 .
$$

Proof of the second part of Theorem B (without the restriction $\alpha \leqq 2$ ). In view of the proof of the first part of Theorem $A$, it is sufficient to consider the case where the points $a_{n k}$ have the property that, for each $r^{\prime}, 0<r^{\prime}<1$, there exists $N$ such that $n>N$ implies $\left|a_{n k}\right|>r^{\prime}$. For convenience, we divide this case further into two sub-cases: (a) $a_{n k}=b_{n k}$, and (b) the contrary case.

Case (a). For obvious reasons, we may assume that, for each sufficiently large $n$, the $n$ points $a_{n k}$ are not only on a diameter, but on a radius, of the unit circle $|z|=1$. Choose $r^{\prime}=1 / 2$. Then, for $n$ sufficiently large,

$$
a_{n k}=r_{n k} e^{i 0_{n}}, \quad 1 / 2<r_{n k}<1, \quad k=1,2, \cdots, n .
$$

Now repeat the argument set forth at the beginning of $\$ 5$ up to the point where a sub-sequence $R_{n_{i}}(z)$ of the sequence $R_{n}^{\prime}(z)$ converges to a limit function $R(z)$, uniformly on any closed point set interior to the unit circle. Here again we take $R_{n}(z)$ for $R_{n_{i}}(z)$. And again we are to prove that, under condition (B), any such limit function $R(z)$ is identically zero.

Suppose that $R(z) \neq 0$. Then there exists $r_{0}, 0<r_{0}<1 / 2$, such that $|R(z)|$ has a positive minimum $m$ on the circle $|z|=r_{0}$, and therefore for $n$ sufficiently large, $\left|R_{n}(z)\right|>m / 2$ on $|z|=r_{0}$.

But, by (3.6), we have

$$
\left|R_{n}(z)\right|<\frac{M}{(1-|z|)^{\alpha}}, \quad M^{2}=\mathfrak{M}_{\alpha}(f), \quad|z|<1 .
$$

Since $R_{n}(z)$ vanishes at $a_{n k}$, we have, by Lemma 6 and the remark following it,

$$
\left|R_{n}\left(r_{0} e^{i \theta_{n}}\right)\right| \leqq \frac{2^{\alpha} M}{\left(1-r_{0}\right)^{2 \alpha}} \prod_{k=1}^{n}\left|\frac{w_{0}-w_{n k}}{1-w_{0} w_{n k}}\right|,
$$

where $0>w_{0}=2 r_{0}-1,0<w_{n \dot{k}}=2 r_{n k}-1$. Set $x_{0}=-w_{0}$. Then the product on the right-hand side of $(10.7)$ is equal to

$$
\frac{1}{P_{n}}=\prod_{k=1}^{n}\left(\frac{x_{0}+w_{n k}}{1+x_{0} w_{n k}}\right)=\prod_{k=1}^{n}\left(1-\frac{\left(1-x_{0}\right)\left(1-w_{n k}\right)}{1+x_{0} w_{n k}}\right) .
$$


Since $-\log (1-h)>h, 0<h<1$, we have

$$
\begin{aligned}
\log P_{n} & >\sum_{k=1}^{n} \frac{\left(1-x_{0}\right)\left(1-w_{n k}\right)}{1+x_{0} w_{n k}}>\frac{1-x_{0}}{1+x_{0}} \sum_{k=1}^{n}\left(1-w_{n k}\right) \\
& =2 \frac{1-x_{0}}{1+x_{0}} \sum_{k=1}^{n}\left(1-r_{n k}\right) .
\end{aligned}
$$

Hence condition (B) implies that $P_{n}$ becomes infinite with $n$, which, in turn, implies that, for $n$ sufficiently large, $\left|R_{n}\left(r_{0} e^{i \theta_{n}}\right)\right|<m / 2$. The contradiction completes the proof for case (a).

Case (b). This case can be reduced to case (a) without difficulty. For, on the one hand, (1.2) transforms (10.6) into

$$
\left|R_{n}^{*}(\zeta)\right|<\left(\frac{1+|c|}{1-|c|}\right)^{\alpha} \frac{M}{(1-|\zeta|)^{\alpha}}, \quad|\zeta|<1,
$$

because

$$
|z|=\left|\frac{\zeta+\lambda \bar{c}}{\lambda+\bar{c} \zeta}\right| \leqq \frac{|\zeta|+|c|}{1+|c||\zeta|}, \quad|\zeta|<1
$$

and

$$
\frac{1}{1-|z|} \leqq \frac{1+|c||\zeta|}{(1-|c|)(1-|\zeta|)} \leqq \frac{1+|c|}{1-|c|} \frac{1}{1-|\zeta|}, \quad|\zeta|<1 .
$$

On the other hand, if condition (B) is valid for the set $a_{n k}$, it is also valid for the equivalent set $b_{n k}$. Hence the argument used in case (a) can be repeated with only formal modifications. The proof is complete.

We remark that Lemma 6 contains a little more than is necessary for the proof of Theorem B. In fact, that lemma enables us to establish the following theorem.

THEOREM $\mathrm{B}^{\prime}$. If $\alpha>1$, and if the set $a_{n k}$ is equivalent to a set $b_{n k}$, such that, for each sufficiently large $n$, the $n$ points $b_{n k}$ are interior to $a \Gamma\left(\theta_{n}\right)$, then a sufficient condition is that

$$
\lim _{n \rightarrow \infty} \prod_{k=1}^{n}\left|2 b_{n k} e^{-i \theta_{n}}-1\right|=0 .
$$

National University of Peking,

Kunming, China. 